\title{
Perceptual Image Quality Assessment Technique for Color Images based on HSI Colour Space
}

\author{
Yildiray Yalman \\ Department of Information Systems Engineering, Piri Reis University, Istanbul, Turkey
}

\begin{tabular}{l}
\hline \hline Article Info \\
\hline Article historys: \\
Received Feb 18, 2020 \\
Revised Dec 10, 2020 \\
Accepted Dec 11, 2020 \\
\hline
\end{tabular}

\section{Keywords:}

HSI colour space

Image quality

Perceptual image quality

Discrete cosine transform

Image histogram

\begin{abstract}
A new full-reference Perceptual Image Quality Assessment (PIQA) technique based on Hue-Saturation-Intensity (HSI) transformation method for colour images is offered in this work. Fundamentally, it is combined with three approaches (colour transformation, histogram processing and Human Visual System (HVS) based weighting) and it uses the Discrete Cosine Transform (DCT) domain. The PIQA is composed of four phases. At the first phase, a colour image is transformed using the HSI because an important characteristic of eyes reaction to intensity of light and colour knowledge is used for quality assessment processes. All channels' DCT coefficients have been calculated at the second phase, because many specifications cannot be assessed in the spatial domain. At the third phase, histogram based quality assessment results are obtained by using histogram of each channel (Hue, Saturation and Intensity). These results are weighted for obtaining perceptual quality result taking into account the HVS specifications at the last phase because the human eye has different sensitivities to the intensity and the others (i.e., hue and saturation). Experimental outcomes about feasibility of the PIQA on test images under different deteriorations, both sensible by the HVS and with the same Peak Signal to Noise Ratio (PSNR) result are offered. The PIQA shows better performance in comparison to state-of-the-art techniques.
\end{abstract}

Copyright () 2020 Institute of Advanced Engineering and Science. All rights reserved.

\section{Corresponding Author:}

Yildiray Yalman,

Department of Information Systems Engineering,

Piri Reis University,

Postane mah., Tuzla, Istanbul 34940, Turkey.

Email: yyalman@pirireis.edu.tr

\section{INTRODUCTION}

Today, digital images are commonly used with application areas ranging from the entertainment industry to the daily life. The Internet, via World Wide Web (www) browsers, thanks to capabilities in computer power and network bandwidth has brought all around the world into offices and homes. In addition, digital images have been frequently used on social media platforms (i.e., instagram, twitter, facebook, etc.) $[1$, 2].

After digital images are acquired, they are generally deteriorated for some signal processing reasons (encoding, compression, acquisition, transmission, steganalysis, manipulations, etc.). From this perspective, quality measurement of distorted or deteriorated images is very important issue at this point. Moreover, it is important that the image quality assessment (IQA) result used for which purpose (forensic or medical issues, human vision system (HVS) based evaluation, etc.) [3]. The amount of assessed deterioration in an image can have dissimilar comments with respect to various practices and defines whether the given image has asked the quality amount to be valuable for implementation [4]. This means that it is very crucial to present implementation oriented IQA techniques.

Basically, there are two kinds of IQA techniques in the literature: No-Reference Image Quality Assessment (NRIQA) techniques and Full-Reference Image Quality Assessment (FRIQA) techniques. 
The NRIQA techniques are known as blind-IQA techniques as there is no way for comparison in between the original visual material and other processed version because original image is not available. Many of the NRIQA algorithms in the literature don't utilize natural image modelling but these algorithms assume that noise type effecting to the image quality is known. Some of them consider image blurring or JPEG compression artefacts by exploring the features of the artefact in different domains [5-6]. These NRIQA techniques are designed to identify special image degradation styles and calculate their occurrence from detailed properties of the categorized artefacts. It can be easily said that the most them can be categorized as distortion-free techniques since they can handle only once or some special distortions $[7,8]$.

In FRIQA techniques, direct assessment in between the original image and its deteriorated model is performed. There is a restriction to the feasibility of FRIQA techniques as the original image is necessary to perform the computation. The basic and well-known FRIQA metrics are Mean Square Error (MSE) and Peak Signal to Noise Ratio (PSNR). These widely-used approaches are generally used in signal processing applications; however the outcomes of these approaches do not relevant enough with the perception of human vision system (HVS) [9]. On the other hand, some other algorithms are used for measuring images, such as PSNR-HVS-M [10], SSIM (Structural SIMilarity) [11], MS-SSIM (MultiScale Structural SIMilarity) [12], UQI (Universal Quality Index) [13], VIF (Visual Information Fidelity) [14] and so on. All of these methods have some problems in terms of reliable HVS based quality assessment. These problems are detailed following sections.

In addition to the fundamental IQA techniques presented above, there is one more inter-type of IQA technique: Reduced-Reference (RRIQA). In fact, it is kind of FRIQA technique. In RRIQA philosophy, only limited information about the original image is accessible while measuring of a digital image under assessment [15-17]. Hence, RRIQA techniques lie in between the NRIQA and FRIQA techniques in terms of accessible information about the original image. All of these just extract some of the specifications from both of them (original one and its deteriorated version) and measures quality of the image corresponding to these specifications, which are the features of all the specifications in the images. Obtained features generally define the image content or deterioration based specifications [18-20].

The presented perceptual IQA technique (PIQA) based on HSI colour model offers a different approach from well-known FRIQA techniques. Basically, the presented technique is combined with three layers (colour transformation, histogram processing and human visual system (HVS) based weighting) and it uses the Discrete Cosine Transform (DCT) domain. Thanks to DCT coefficients, high and low frequency values can be easily decomposed and then low frequency values are used for measurement processes because high frequency values can be ignored while image quality calculation in terms of the HVS.

The paper is arranged as follows. HSI colour model, the DCT and histograms of images are introduced in Section 2. The phases of the presented technique are detailed in Section 3. Section 4 presents experimental IQA results and comparisons. Conclusions are provided in Section 5.

\section{SOME FUNDAMENTAL THEMES}

HSI colour space, the DCT and image histogram have been used for the presented HVS based PIQA technique. Their details and usage fundamentals have been presented below.

\subsection{HSI colour transformation}

In the RGB (red, green, blue) colour space, each channel appears in its main spectral parts of colour image. It is fundamentally based on a Cartesian coordinate system. The term full-colour image is used as a 24bits ( 8 bits for each colour channel) RGB colour image (Figure 1a). This model is suitable for colour presentation (as in image captured by any camera or mobile device in a display system) [21].

On the other hand, when human observes a colour object, it is described by its hue, saturation and brightness. Hue $(\mathrm{H})$ is a colour feature that defines a pure colour. Saturation $(\mathrm{S})$ presents a measurement of the grades to which a pure color is subtilized by white light. Brightness is a subjective descriptor and it objectifies the achromatic concept of intensity (I) and it is a key feature in defining colour perception [1,21]. This is called as HSI colour model (Figure 1b). The HSI decouples the intensity part from the color carrying information (Hue, Saturation) in a digital color image [21]. From this point of view, it can be said that the HSI model is an important transformation method for improving an IQA technique based on the HVS. The HSI colour space model has been used for the presented PIQA technique because of this important feature.

Given an image in RGB colour type, H, S and I channels can be calculated using the Eq. (1), Eq. (3) and Eq. (4), respectively. 


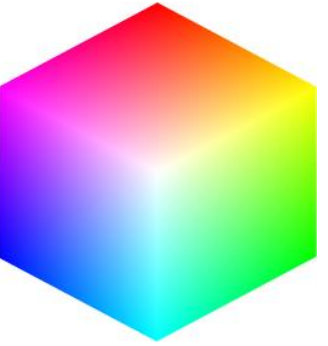

(a)

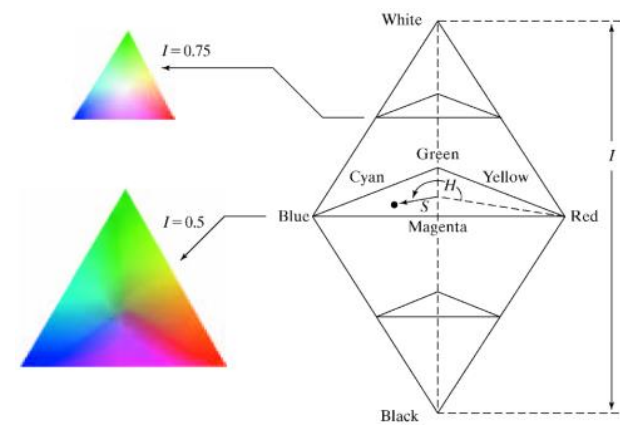

(b)

Figure 1. Color spaces (RGB colour cube (a), triangular HSI colour space (b))

$$
H=\left\{\begin{array}{ccc}
\theta & \text { if } & B \leq G \\
360-\theta & \text { if } & B>G
\end{array}\right.
$$

with

$$
\begin{aligned}
& \theta=\cos ^{-1}\left\{\frac{\frac{1}{2}[(R-G)+(R-B)]}{\left[(R-G)^{2}+(R-B)(G-B)^{1 / 2}\right]}\right\} \\
& S=1-\frac{3}{R+G+B}[\min (R, G, B)] \\
& I=\frac{1}{3}(R+G+B)
\end{aligned}
$$

Well-known Lena images (in RGB form), its distorted (blurring) version and their Hue, Saturation and Intensity channels can be seen in Figure 2. In addition, deteriorations or differences can be easily sensed by the HVS on all channels, too.

It is assumed that the RGB values are normalized to the range between 0 and 1 . Angle $\Theta$ is calculated with respect to the red axis of the HSI. Hue can be normalized to the range between 0 and 1 dividing by $360^{\circ}$ all values resulting from Eq. (1). Other parts (i.e., S and I) are in this range if the given RGB values are in interval $[0,1]$.

The HSI colour space gives us an opportunity to describe colours in terms of more readily understandable. The intensity referred is the brightness and the hue is what we normally think of as "colour". The saturation is a measure of how much white is in a colour; for example, pink is red with more white, so it is less saturated then a pure red. People can concern to this methodology of defining colour, for example "a deep, bright orange" would have a large intensity ("bright"), a hue of ("orange") and a high value of saturation ("deep"). People can imagine a colour in their minds, but if the colour is defined in terms of its RGB colour space (for example, $\mathrm{R}=240, \mathrm{G}=100, \mathrm{~B}=25$ ), most people would have no idea how this colours view. Thus, the HSI colour model has been improved based on relating to the HVS [1].

A significant specification of the HVS is its distinct reaction to the intensity of the light and colours thoroughly concerned to the natural specifications of the human eye [22-24], which are used and reflected into the presented technique with varied percentages.

\subsection{Discrete cosine transform}

One of the most famous transformation methods is Discrete Cosine Transform (DCT) and is generally utilized for image compression. The DCT like Fourier transform uses sinusoidal functions. The main distinction is that the DCT based functions are not complex; it uses only cosine functions not sine functions. The 2D-DCT equation for $\mathrm{N} \times \mathrm{N}$ image is given by

$$
C(u)=\alpha(u) \alpha(v) \sum_{r=0}^{N-1} \sum_{c=0}^{N-1} I(r, c) \cos \left[\frac{(2 r+1) u \pi}{2 N}\right] \cos \left[\frac{(2 c+1) v \pi}{2 N}\right]
$$

where

$$
\alpha(u), \alpha(v)=\left\{\begin{array}{ccc}
\sqrt{\frac{1}{\mathrm{~N}}} & \text { for } & u, v=0 \\
\sqrt{\frac{2}{\mathrm{~N}}} & \text { for } & u, v=1,2, \ldots, N-1
\end{array}\right.
$$




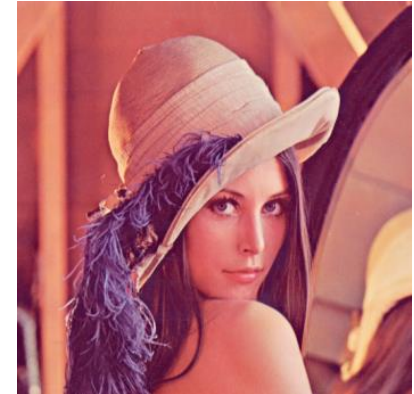

(a)

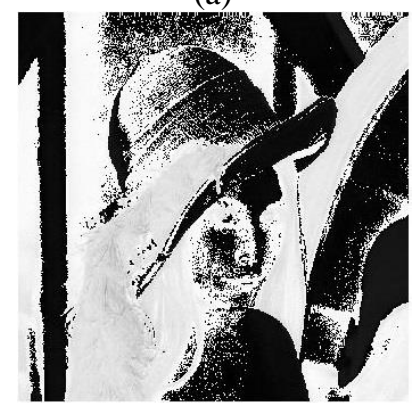

(b)

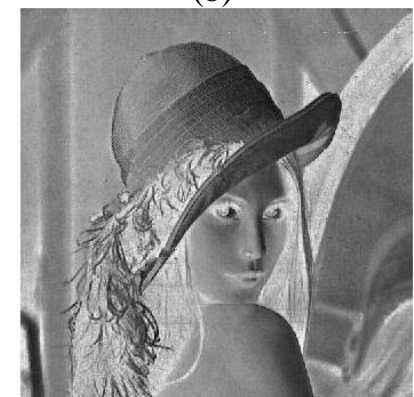

(c)

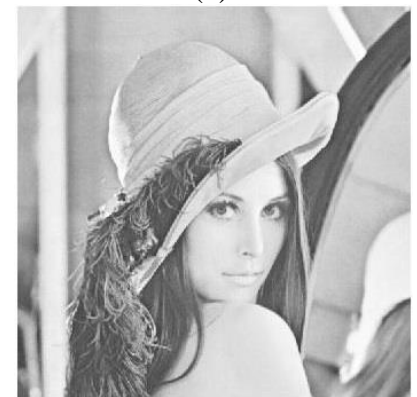

(d)

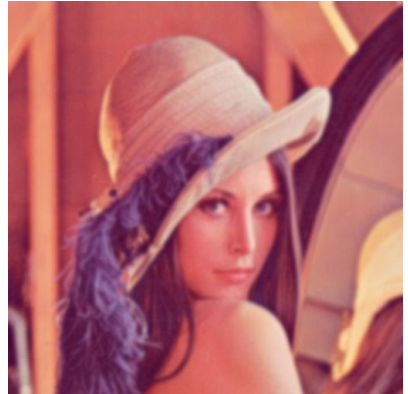

(e)

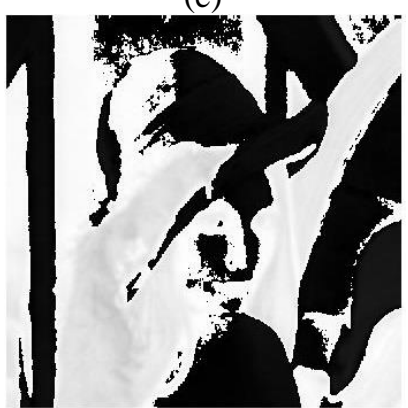

(f)

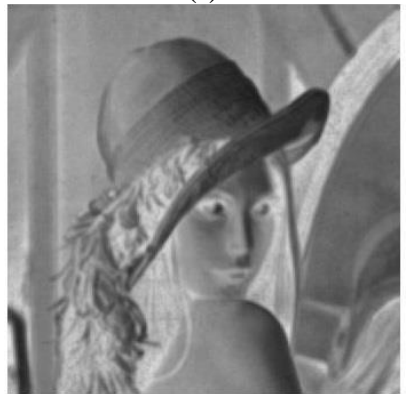

(g)

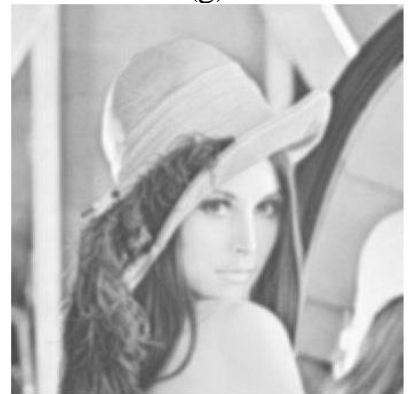

(h)

Figure 2. Images and HSI channels (An original RGB image, Hue, Saturation and Intensity channels of the RGB image (b, c, d), a distorted version of the RGB image (the PSNR result is $27.67 \mathrm{~dB}$ ) (e), Hue, Saturation and Intensity channels of the distorted RGB image (f, g, h)).

Figure 3 shows intensity component obtained after the HSI transformation of blurred Lena and its DCT version. The DCT coefficients can be divided to main regions as Low Frequency (DC) and High Frequency (AC). DC coefficients are more important than the AC coefficients for the HVS thanks to AC coefficients contains deep details. In contrast to this situation, the DC coefficients have general characteristics of the image. From this point of view, the first quarter part of the DCT coefficients is used for the PIQA calculation. 

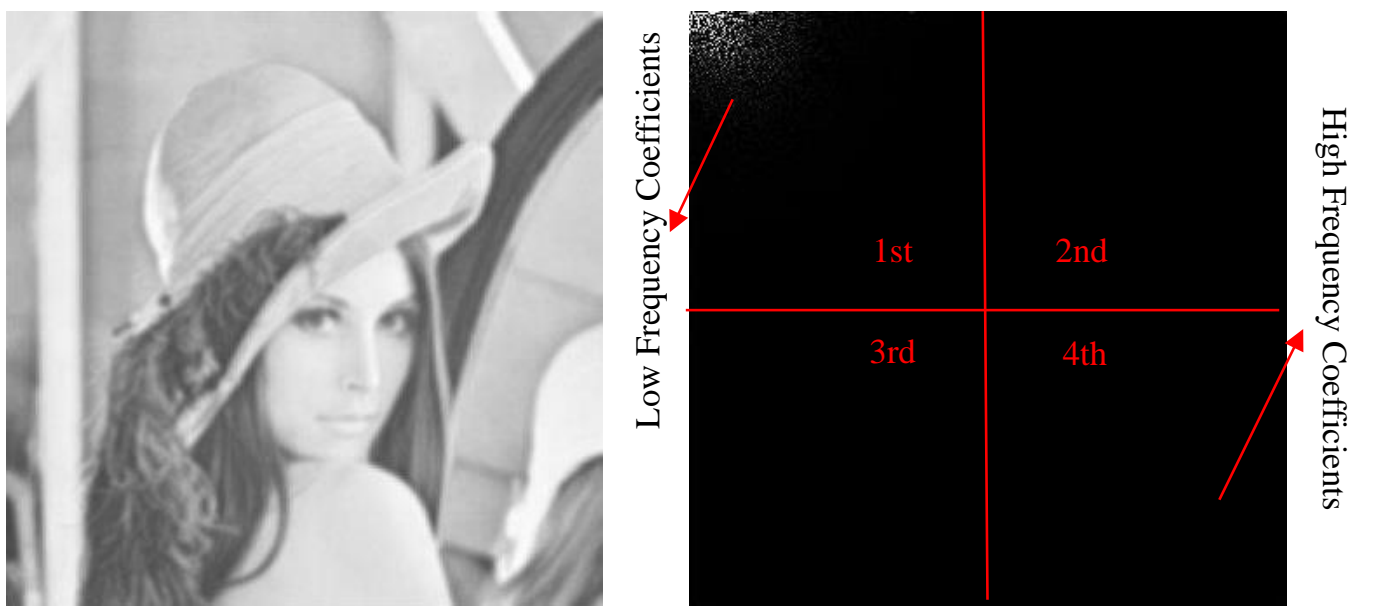

Figure 3. Intensity channel and its DCT version (Intensity component (a) and its DCT version and quarters (b)).

\subsection{Digital image histogram}

The histogram of an image with intensity levels in the range [0,L-1] is a discrete function $H\left(v_{k}\right)=n_{k}$, where $v_{k}$ is the $k$ th intensity value and $n_{k}$ is the number of pixels in the image with intensity $v_{k}$. It is general implementation to normalize a histogram by dividing each of its parts by all number of pixels in the image, defined by the product $R C$, where, as usual, $R$ and $C$ are row and column dimensions of the image. Therefore, a normalized histogram is written by $p\left(v_{k}\right)=n_{k} / R C$, for $k=0,1,2, \ldots, L-1 . p\left(v_{k}\right)$ is an estimate of the probability of occurrence of intensity grade $v_{k}$ in an image. The sum of all parts of a normalized histogram is equal to 1 [21].

Not only image pixel values but also whole number clusters can be depicted with a histogram. From this general concept, the first quarter part of the DCT coefficients of each HSI channel is used to obtain histograms in this presented study. This approach is an important aspect of the PIQA.

Distorted versions of the original Lena images and DCT values based H, S and I histograms are given in Figure 4 and Figure 5, respectively.

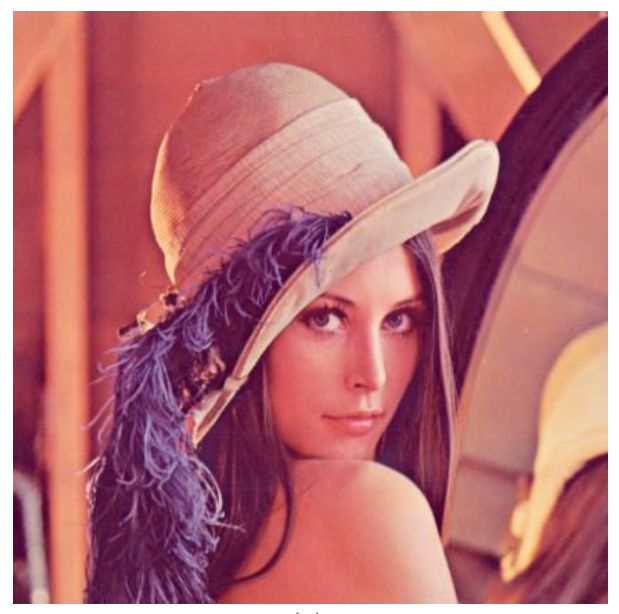

(a)

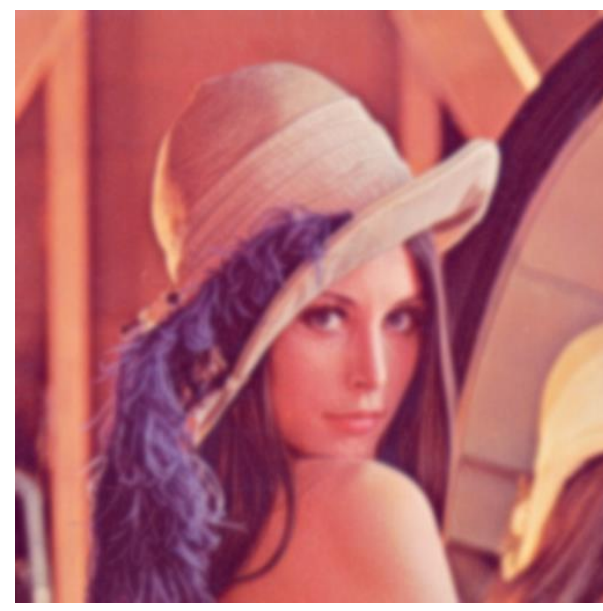

(b)

Figure 4. Test Images (Original Lena image (a) and its blurred version (PSNR is $27.67 \mathrm{~dB}$ (b)).

Although, it is thought to be image qualities are the same considering the PSNR results (i.e., 27.67 $\mathrm{dB}$ ), it can be viewed that this is not really true considering the histograms depicted after DCT (Figure 5). H and $\mathrm{S}$ histograms have been obtained after normalization processes because $\mathrm{H}$ and $\mathrm{S}$ channels can be contain floating point numbers which are out of range. Thus, normalization process has been realized to adjust the values between $[0,255]$.

Dissimilarities between the original image's histogram and its deteriorated version's histogram are given in Figure 5. Thus, this situation has been main motivation to develop the PIQA technique presented in this study. 


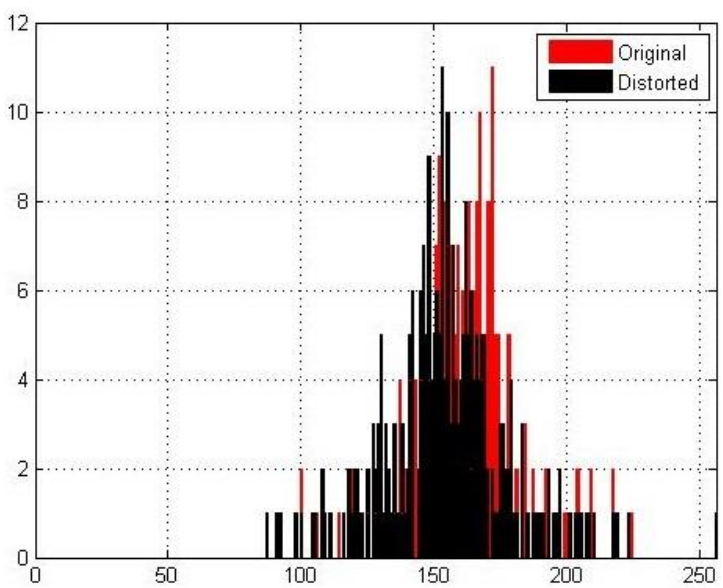

(a)

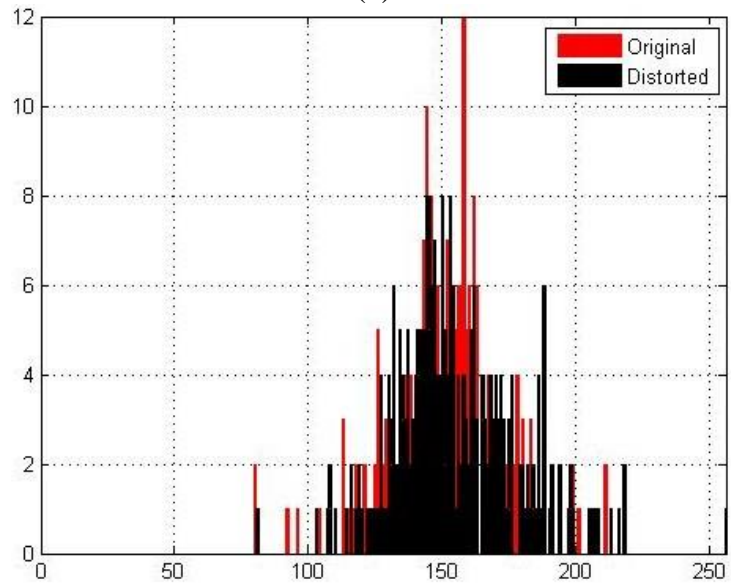

(b)

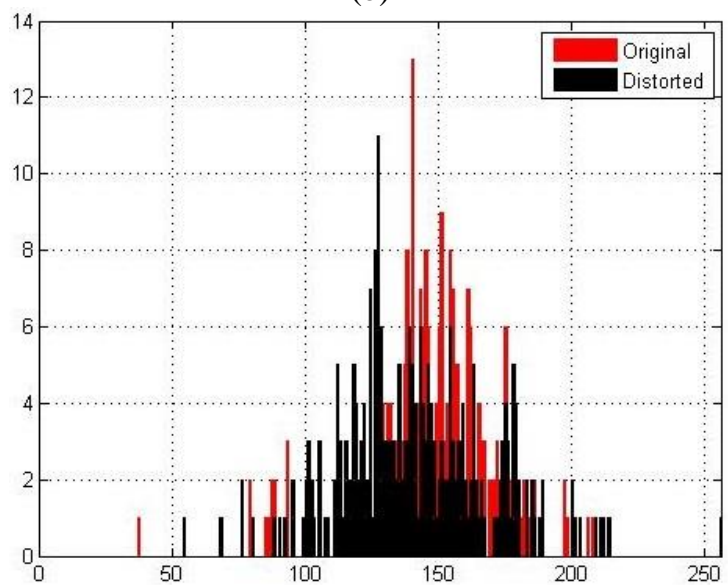

(c)

Figure 5. The DCT coefficients' Histograms for original image and its distorted version depicted in Fig. 4 (Histograms of the Hue component (a), Histograms of the Saturation component (b), Histograms of the Intensity component (c)).

\section{THE PROPOSED PERCEPTUAL IMAGE QUALITY ASSESSMENT TECHNIQUE (PIQA)}

The developed PIQA technique, with the steps of its four basic phases, i.e. the HSI color space, the DCT based Histograms computation for all HSI components, obtaining histograms of their first quarter parts'

( $\boldsymbol{H}$ and $\hat{\mathcal{H}}$ ) and weighting of these considering the human perception are detailed. The basic flow of the PIQA method is given in Figure 6. 


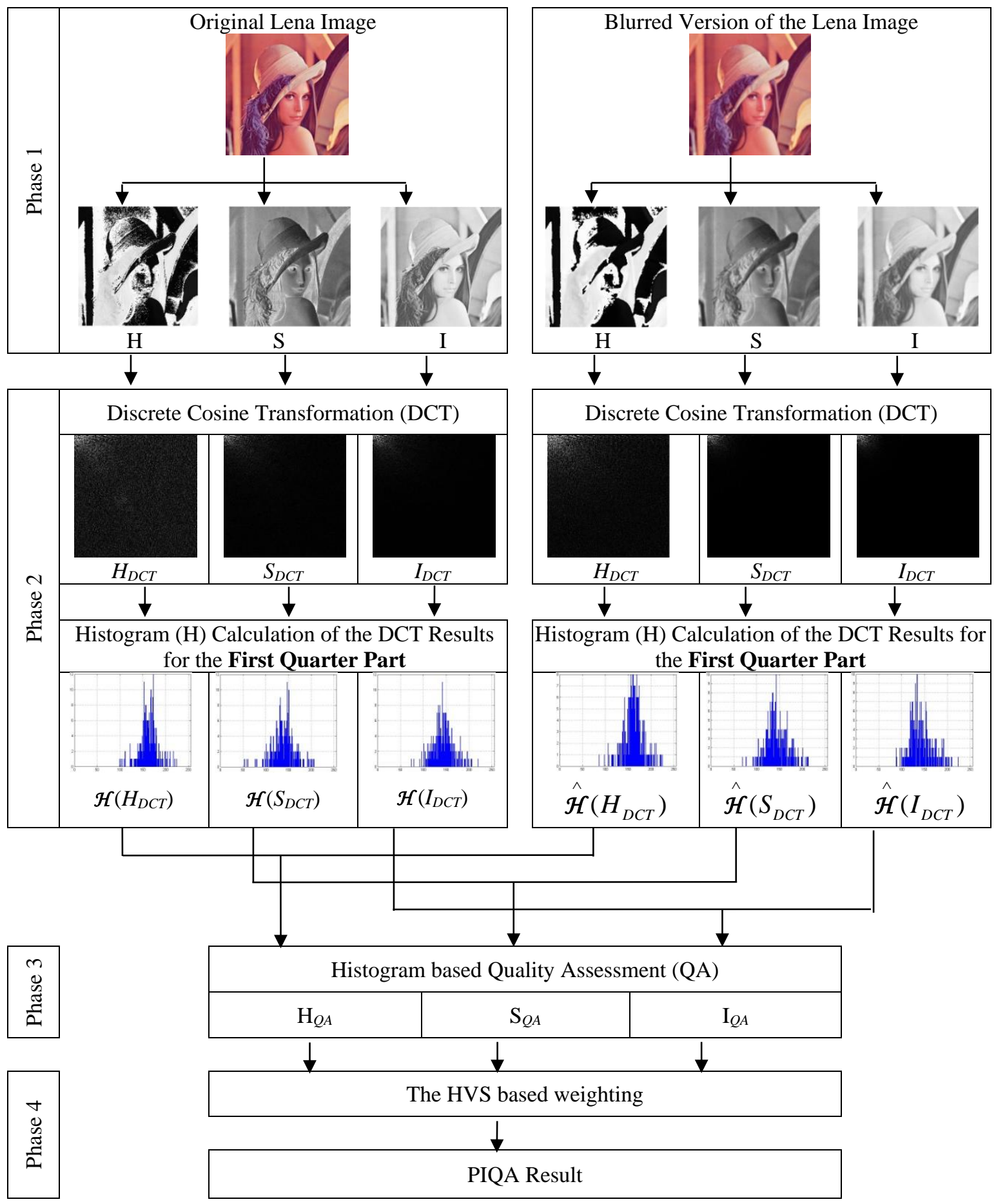

Figure 6. Block scheme of the presented PIQA technique

\subsection{Steps of the proposed PIQA technique}

The presented PIQA technique's assessment result is obtained in four phases as depicted above. Each phase is listed and clarified below:

- Phase 1: As it is previously described, intensity information and color information should be separated from the color image because human eye response is different for each of them [23, 24, 25]. Thus, HSI transformation equations given in Eq. (1-4) are realized on color images (original and distorted versions) at the first phase. Those equations are utilized when RGB to HSI conversion where I channel refers to intensity while $\mathrm{H}$ and $\mathrm{S}$ refer to the hue and saturation knowledge in an image. $\mathrm{H}$ and S channels are very important parts of the HSI model because they are decisive for color 
determination on this transformation model. The HSI channels' values are used and reflected into the presented technique with different percentages as explained following phases.

- Phase 2: This phase has two main sequential parts: DCT calculation and histogram achieving. At the first part, the DCT coefficients are calculated for each HSI channels (i.e., $H_{D C T}, S_{D C T}$ and $I_{D C T}$ ). Therefore, high and low frequency information can be separated.

The histogram of an image with intensity values in the range $[0, V-1]$ is a discrete function $h\left(m_{k}\right)=n_{k}$, where $m_{k}$ is the $k$ th intensity value and $n_{\mathrm{k}}$ is the number of pixel in the image with intensity $m_{k}$. Histogram $(\mathrm{H})$ calculation of the DCT coefficients is realized for its first quarter part at the second part (i.e., $\mathrm{H}\left(H_{D C T}\right), \mathrm{H}\left(S_{D C T}\right)$ and $\left.\mathrm{H}\left(I_{D C T}\right)\right)$ because the HVS cannot be easily detected deterioration on the high frequency components. These parts are performed for distorted image, too (i.e., $\left.\hat{\mathcal{H}}\left(H_{D C T}\right), \hat{\mathcal{H}}\left(S_{D C T}\right), \hat{\mathcal{H}}\left(I_{D C T}\right)\right)$ as seen in Fig. 6.

- $\quad$ Phase 3: The histogram quality results $\left(\mathrm{H}_{\mathrm{QA}}, \mathrm{S}_{\mathrm{QA}}, \mathrm{I}_{\mathrm{QA}}\right)$ are calculated for each channel at this phase. While this step is achieved on the first part of the DCT coefficients' histograms, histogram based comparing approach is used. The Quality Assessment (QA) is composed of two parts and it is defined as:

$\mathrm{H}_{\mathrm{QA}}=\Delta_{\mathrm{TA}} \mathrm{F} \times \mathrm{D}$

The first part of $\mathrm{H}_{\mathrm{QA}}$ is $\Delta_{\mathrm{TA}} \mathrm{F}$ (interhistogram difference factor). Histograms $(\mathrm{H})$ are achieved from the original HSI image's channel (i.e., $\mathrm{H}\left(H_{D C T}\right)$ ) and the deteriorated HSI image's channel (i.e., $\left.\mathcal{H}\left(H_{D C T}\right)\right)$ are realized to the subtraction operation to find the difference as seen below:

$$
\Delta=\left|\mathcal{H}\left(H_{D C T}\right)-\hat{\mathcal{H}}\left(H_{D C T}\right)\right|
$$

The $\Delta$ is a difference vector between test image and its deteriorated histogram version. Total alteration $\left(\Delta_{\mathrm{TA}}\right)$ between them is achieved by summation of all indices of the $\Delta$ (Eq. 9) and $0 \leq \Delta \leq 2 \times R \times C$. In this equation, $R$ and $C$ refers row and column numbers, respectively.

$$
\Delta_{T A}=\sum_{i=1}^{256} \Delta_{i}
$$

Then, $\Delta_{\mathrm{TA}} \mathrm{F}$ varying between 0 (worst) and 1 (best) is obtained using $\Delta_{\mathrm{TA}}$ as given in Eq. (10) [26].

$$
\Delta_{T A} F=1-\left(\frac{\Delta_{T A}}{\Delta_{T A_{\max }}}\right)
$$

$\Delta_{T A_{\max }}$ refers that the top value on the number of histogram alteration is $(2 \times R \times C)$. The range of $\Delta_{\mathrm{TA}} \mathrm{F}$ is $[0,1]$. The best value 1 can be calculated if $\mathcal{H}\left(H_{D C T}\right)=\hat{\mathcal{H}}\left(H_{D C T}\right)$.

The second part of the $\mathrm{H}_{Q A}$ (Eq. 7) is the $D$ (histogram Distortion) and it is used to obtain correlation quality between $\mathcal{H}\left(H_{D C T}\right)$ and $\hat{H}\left(H_{D C T}\right)$. It is obtained by using Eq. (11) [26].

$$
D=\frac{\sum_{j}\left(\mathcal{H}\left(H_{D C T}\right)_{j} \times \hat{\mathcal{H}}\left(H_{D C T}\right)_{j}\right)}{\sum_{j}\left(\mathcal{H}\left(H_{D C T}\right)_{j}\right)^{2}}
$$

Like the $\Delta_{\mathrm{TA}} \mathrm{F}$, the best $\mathrm{D}$ value is also 1 , which is calculated when $\mathcal{H}\left(H_{D C T}\right)=\hat{\mathcal{H}}\left(H_{D C T}\right)$. The expressions above prove that the top $\mathrm{H}_{Q A}$ number is 1 , which results when $\Delta_{\mathrm{TA}} \mathrm{F}$ and $\mathrm{D}$ numbers are equal to 1 . The worst number of the $\mathrm{H}_{Q A}$ is 0 , which occurs when $\Delta_{\mathrm{TA}}=\Delta_{T A_{\max }}$ [8]. These processes are sequentially realized to the other channels of the HSI image (i.e., $\mathcal{H}\left(\mathrm{S}_{D C T}\right), \mathcal{H}\left(I_{D C T}\right)$ and their distorted images' versions) and then, $\mathrm{S}_{Q A}$, and $\mathrm{I}_{Q A}$ are calculated.

- Phase-4: This phase is the last step of the PIQA technique. The QA results $\left(\mathrm{H}_{Q A}, \mathrm{~S}_{Q A}\right.$, and $\left.\mathrm{I}_{Q A}\right)$ which calculated for H, S and I are weighted by considering the HVS features. These features are too significant for perceptual specification of the presented PIQA technique.

Figure 7a shows the important components of the human eye-the cornea, iris, lens and retina. The structure of the retina, the mat of photoreceptors at the back of the eye, does differ in two groups. 
Cephalopod photoreceptors are built on the same rhabdomeric plan as those of the arthropods, but vertebrate receptors are of the ciliary type, in which the layers of membrane containing light-sensitive pigment are stacked in an outer segment of the cell. There are two types of ciliary photoreceptor, rods and cones (Figure 7b) [24].

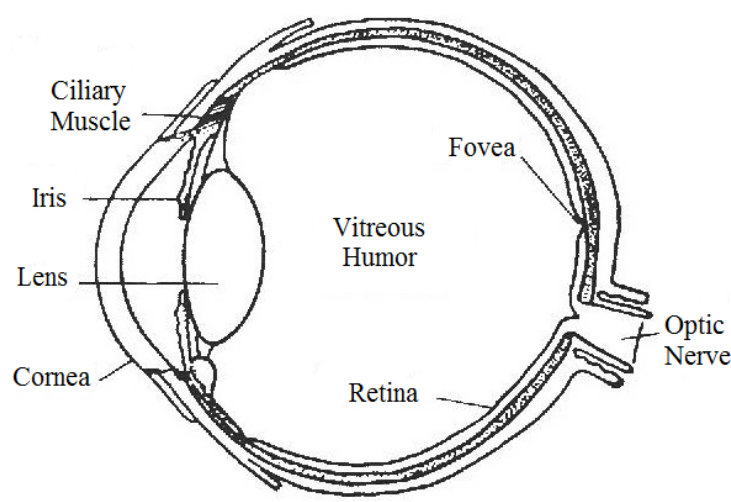

(a)

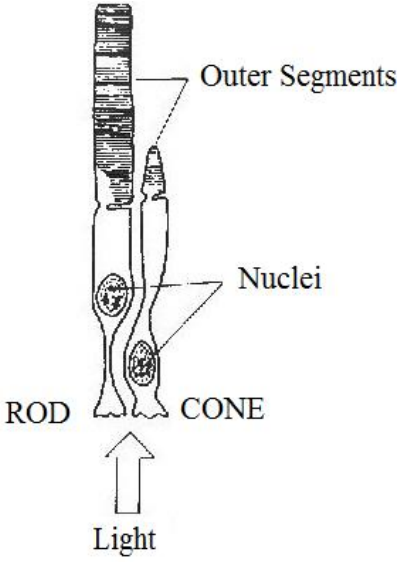

(b)

Fig. 7. Human eye structure (Human eye (a), a rod cell and a cone cell in the vertebrate retina (b)).

The fovea part of the human eye contains cone cells and rod cells. The rod cells are responsible for vision at especially low light levels. The rod cells don't ensure color vision and have no effect on spatial perception $[4,27,28]$. On the contrary, the cone cells are responsible for vision at higher light levels and spatial perception. Rod cells' density is $200,000 \mathrm{rods} / \mathrm{mm}^{2}$ (120 million) and cone cells' density is 150,000 cones $/ \mathrm{mm}^{2}$ (7 million) [21, 29, 30]. The corollary of this case, the proportion of the Rods $\left(R_{P}\right)$ and the proportion of the Cones $\left(C_{P}\right)$ can be easily obtained as seen below:

$C_{P}=7 \times 10^{6} /\left(120 \times 10^{6}+7 \times 10^{6}\right)=0.0551$,

$R_{P}=120 \times 10^{6} /\left(120 \times 10^{6}+7 \times 10^{6}\right)=0.9449$.

At the end of the all processes, the PIQA result can be calculated using Eq. (14).

$$
P I Q A=\left(I_{Q A} \times R_{P}\right)+\left(\frac{H_{Q A}+S_{Q A}}{2}\right) \times C_{P}
$$

Thanks to $C_{P}$ and $R_{P}$, the HVS specifications are taken into account and more suitable results with human perception can be clearly obtained as detailed in the next section.

\section{EXPERIMENTAL RESULTS}

Generally used Lena reference image and its six deteriorated samples have been used for experimental applications and performance evaluations at the first step. It is distorted with well-known deterioration methods to yield the same Peak Signal to Noise Ratio (PSNR) values relative to the original Lena image.

The PIQA and the PSNR values for all of the images $(512 \times 512 \times 3)$ are listed in Figure 8 . It can be said that the PSNR results of the Figure $8 \mathrm{~b}, 8 \mathrm{c}, 8 \mathrm{~d}, 8 \mathrm{e}, 8 \mathrm{f}$ and $8 \mathrm{~g}$ are $27.67 \mathrm{~dB}$. This means that these deteriorations affected in the same way to the original test image contrary to the fact based on at least basic perceptual evaluation. In addition, it can be said that classical PSNR measure cannot differentiate all probable diversities between test image and its deteriorated versions [22]. But the proposed PIQA technique can easily differentiate and sort (i.e, from 0.4389 to 0.8537 ) them. This means that Figure $8 \mathrm{~g}$ (blurred version) is worse than Figure 8a (sharpen) in terms of the HVS.

In addition to the PSNR, there are some well-known image quality assessment techniques such as PSNR-HVS-M [10], MS-SSIM [11], SSIM [12], UQI [13] in order to compare the presented PIQA technique (Table 1).

Considering the other IQA techniques mentioned above, it can be said that their results can differentiate to the images like the presented PIQA technique, with respect to the PSNR. But, the PSNR-HVS$\mathrm{M}$ result implies that Figure $8 \mathrm{~d}$ is better than Figure 8b. In addition, the MS-SSIM results are different from each other but according to this technique's results, Fig. $8 \mathrm{~g}$ has better quality than Figure 8c, Figure 8d and Figure 8f. This means that it cannot sort to distorted images according to the HVS, too. On the other hand, the SSIM and UQI quality assessment values verified to be false, as they indicate that the image quality for Figure $8 \mathrm{~g}$ is better than that of Figure 8c. Thus, it can be said that these results are not compatible with the HVS, too. 


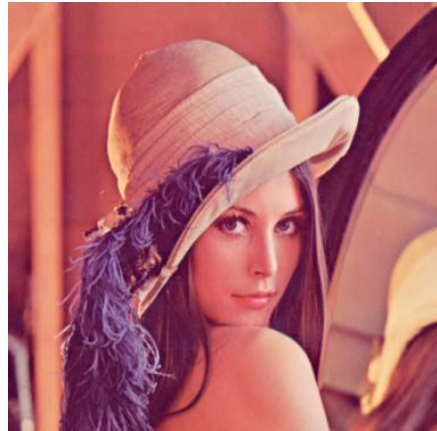

(a)

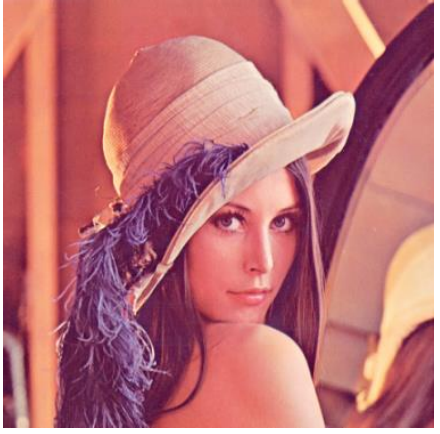

(b)

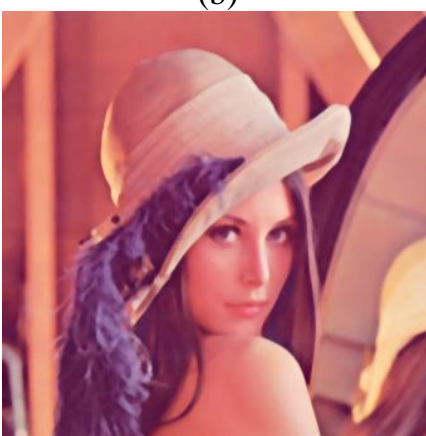

(f)

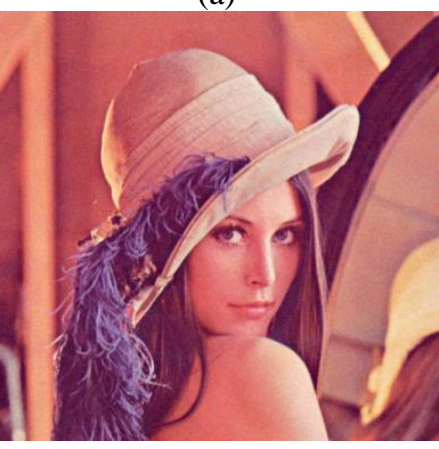

(c)

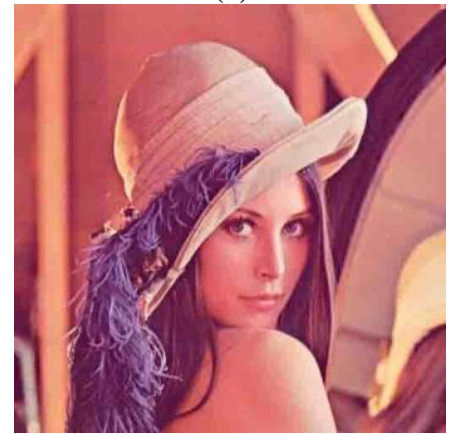

(e)

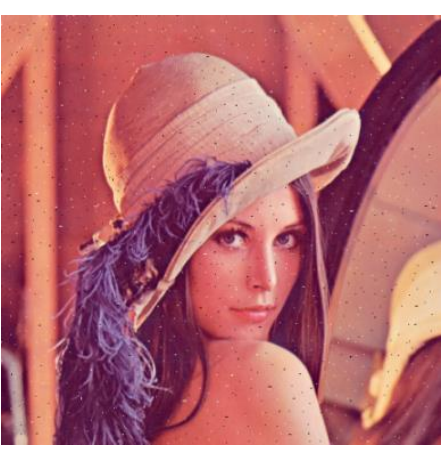

(d)

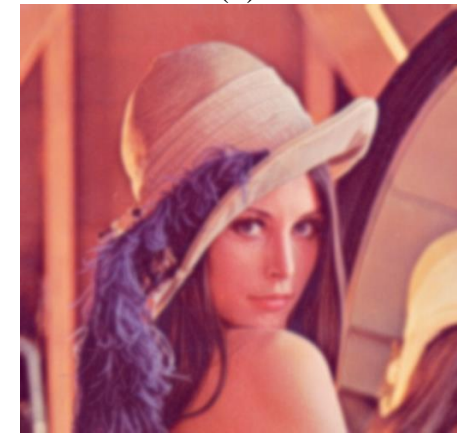

(g)

Figure 8. Experimental results for Lena test image (all deteriorated images' PSNR $=27.67 \mathrm{~dB})($ Original Lena image (a), Sharpen (PIQA = 0.8537) (b), Gaussian (PIQA = 0.8516) (c), Salt \& pepper noise (PIQA = $0.8179)(\mathrm{d})$, Median $(\mathrm{PIQA}=0.7755)(\mathrm{e}), \mathrm{JPEG}$ compression $(\mathrm{PIQA}=0.7361)(\mathrm{f})$, Blurring $(\mathrm{PIQA}=0.4389)$ $(\mathrm{g}))$.

Table 1. Experimental results of the PIQA and other well-known IQA methods.

\begin{tabular}{llcccccc}
\hline Test Image & Distortion & $\begin{array}{c}\text { PSNR } \\
(\mathrm{dB})\end{array}$ & PSNR-HVS-M $(\mathrm{dB})$ & MS-SSIM & SSIM & UQI & PIQA \\
\hline Fig. 8b & Sharpen & 27.67 & 25.0338 & 0.9905 & 0.9097 & 0.8698 & $\mathbf{0 . 8 5 3 7}$ \\
Fig. 8c & Gaussian & 27.67 & 29.0506 & 0.9521 & 0.6285 & 0.5257 & $\mathbf{0 . 8 5 1 6}$ \\
Fig. 8d & Salt \& Pepper & 27.67 & 29.5427 & 0.9509 & 0.8640 & 0.8204 & $\mathbf{0 . 8 1 7 9}$ \\
Fig. 8e & Median & 27.67 & 24.3752 & 0.9546 & 0.7683 & 0.4989 & $\mathbf{0 . 7 7 5 5}$ \\
Fig. 8f & Jpeg & 27.67 & 24.7130 & 0.9402 & 0.7781 & 0.5034 & $\mathbf{0 . 7 3 6 1}$ \\
Fig. 8g & Blurring & 27.67 & 23.9838 & 0.9546 & 0.7839 & 0.6147 & $\mathbf{0 . 4 3 8 9}$ \\
\hline
\end{tabular}

The other test image (Baboon) and its four deteriorated versions (under Gaussian noise (mean 0 and variance $\left(\sigma^{2}\right)$ ) are utilized for another experiment (Figure 9). 


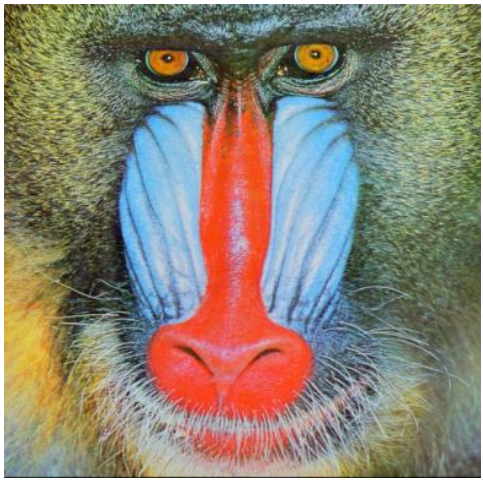

(a) Original

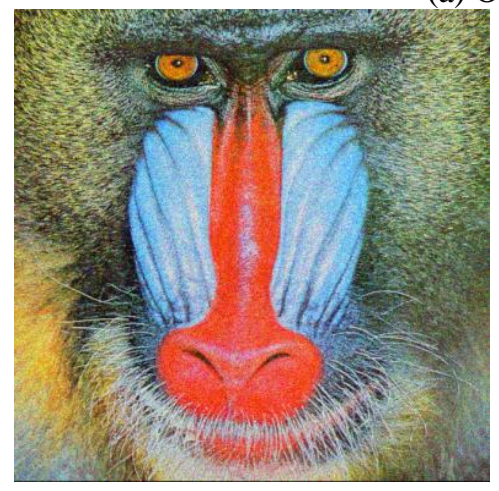

(b) $\sigma^{2}=0.01$

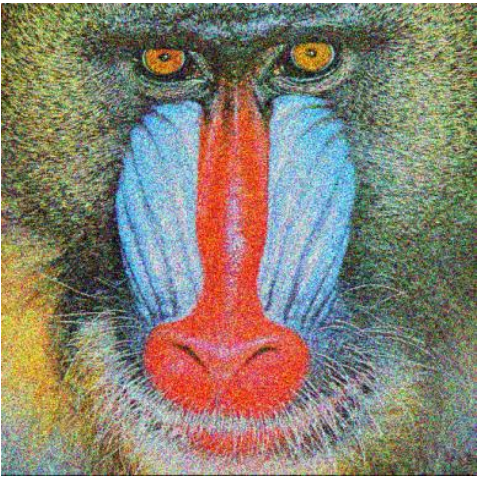

(d) $\sigma^{2}=0.05$

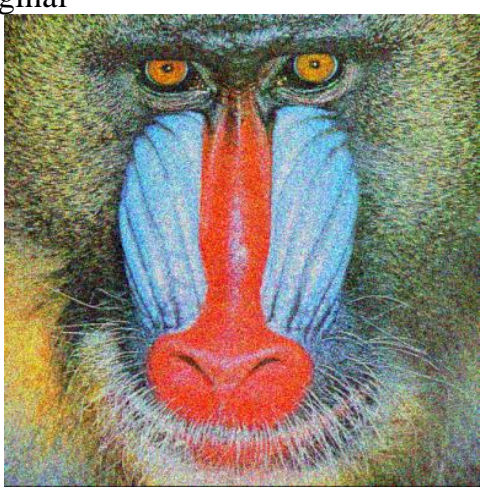

(c) $\sigma^{2}=0.03$

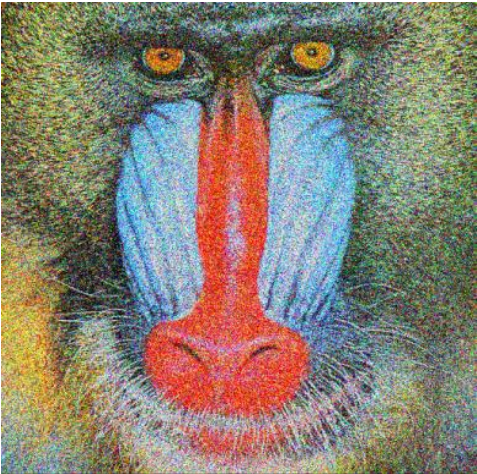

(e) $\sigma^{2}=0.07$

Figure 9. Baboon image and its deteriorated versions under Gaussian noise.

Figure 10a shows the PSNR and the PSNR-HVS-M IQA results. Similarly, other counterparts' and the PIQA results are given in Figure 10b. The figures depicts that while the other IQA methods' results characteristics are seen as exponential distribution, the PIQA technique's results are observed as different. For example, all other methods' results mentioned above imply that Figure 9c better than Figure 9d. However the PIQA results imply just the opposite because the PIQA technique takes into account the HVS characteristics.

The calculation or computation speed is also a significant criterion in the most applications, such as the IQA while working on image data bases [4]. The experimental studies have been realized on Intel® Core i7 $5500 \mathrm{CPU}$ running at $2.4 \mathrm{GHz}$ with $8 \mathrm{~GB}$ RAM, and the PIQA were implemented in Matlab®. As seen in Table 2, the PIQA is about 25 times faster than the PSNR-HVS-M and better than the other techniques.

In addition to some valuable conclusions mentioned above, 24 test images downloaded from [31] shown in Figure 11 have been used for other applications in order to measure the PIQA technique. All images were coded by Least Significant Bit (LSB) steganography algorithm (1 bit per pixel). Thus, sensitivity and the capability of differentiation of the IQA techniques have been measured, too. 


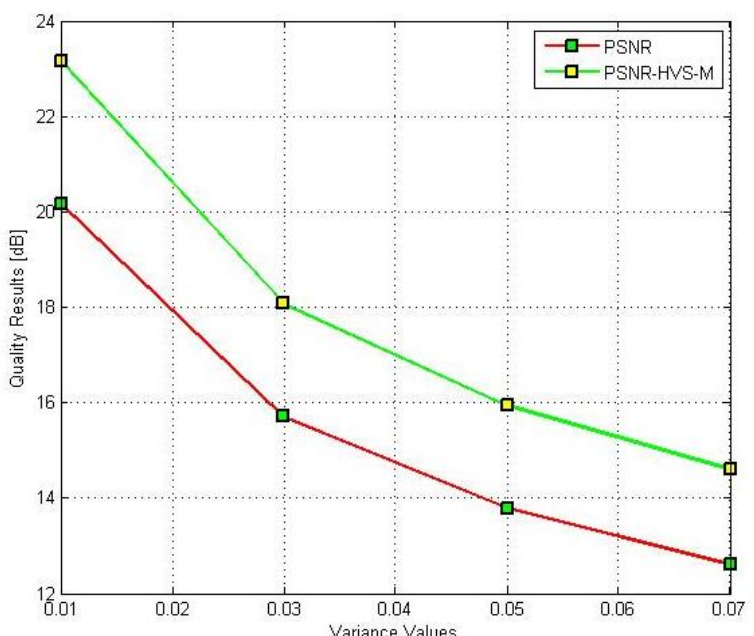

(a)

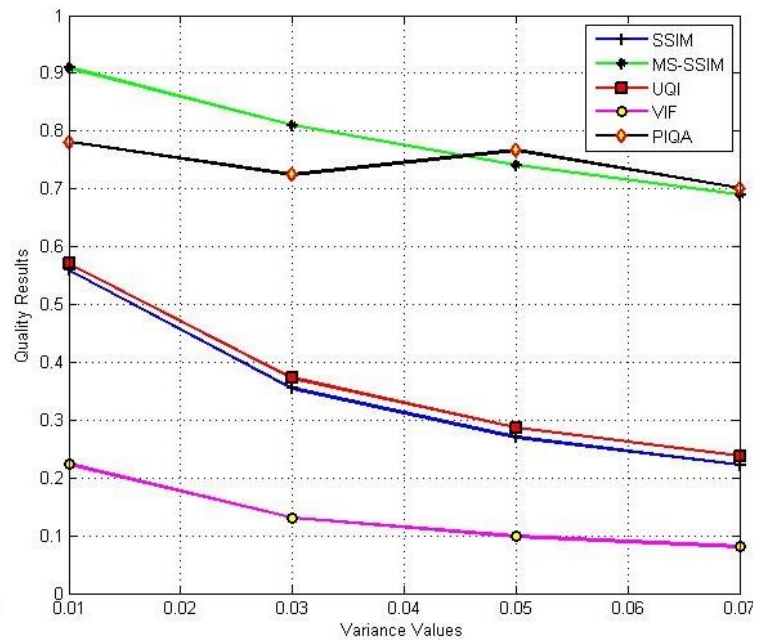

(b)

Figure 10. Experimental results of the IQA techniques' results for Baboon image under speckle noise (mean 0 and variance $\sigma^{2}$ ) (PSNR and PSNR-HVS-M results (a), SSIM, MS-SSIM, UQI, VIF and PIQA results (b)).

Table 2. Processing times of IQA techniques for Fig. 8a and Fig. 8g.

\begin{tabular}{lc}
\hline IQA Methods & Processing Time $(\mathrm{sec})$ \\
\hline PSNR-HVS-M & 8.9376 \\
MS-SSIM & 0.3862 \\
UQI & 0.3627 \\
SSIM & 0.3542 \\
PIQA & $\mathbf{0 . 3 4 9 1}$ \\
\hline
\end{tabular}

The PSNR results are the same (i.e., $57.67 \mathrm{~dB}$ ) for all images as seen in Table 3. In addition, the MSSSIM and the SSIM results are the same. This means that these techniques cannot differentiate images because the quality results are the same. Moreover, the PSNR-HVS-M and the UQI methods generate repeatable and close IQA results. But, the presented PIQA technique can differentiate in terms of visual effects of low distortions on images. The PIQA results are between 0.4830 and 0.9740 as seen in Table 3 .

Table 3. IQA results of test images coded by LSB (1 bits per pixel).

\begin{tabular}{|c|c|c|c|c|c|c|}
\hline Test Image & $\begin{array}{c}\text { PSNR } \\
(\mathrm{dB})\end{array}$ & $\begin{array}{c}\text { PSNR-HVS-M } \\
\text { (dB) }\end{array}$ & MS-SSIM & SSIM & UQI & PIQA \\
\hline $\mathrm{k} 01^{\square}$ & 57.67 & 58.39 & 0.999 & 0.999 & 0.998 & 0.7306 \\
\hline $\mathrm{k} 02^{\text {ㅁ }}$ & 57.67 & 58.42 & 0.999 & 0.999 & 0.998 & 0.5261 \\
\hline${\mathrm{k} 03^{\square}}^{\square}$ & 57.67 & 58.33 & 0.999 & 0.999 & 0.984 & 0.7149 \\
\hline $\mathrm{k} 04$ & 57.67 & 58.50 & 0.999 & 0.999 & 0.996 & 0.4830 \\
\hline $\mathrm{k} 05^{\square}$ & 57.67 & 58.37 & 0.999 & 0.999 & 0.999 & 0.8540 \\
\hline $\mathrm{k} 06^{\square}$ & 57.67 & 58.36 & 0.999 & 0.999 & 0.995 & 0.7050 \\
\hline $\mathrm{k} 07^{\text {ㅁ }}$ & 57.67 & 58.35 & 0.999 & 0.999 & 0.991 & 0.8083 \\
\hline $\mathrm{k} 08^{\square}$ & 57.67 & 58.37 & 0.999 & 0.999 & 0.999 & 0.6083 \\
\hline k09" & 57.67 & 58.28 & 0.999 & 0.999 & 0.993 & 0.9740 \\
\hline $\mathrm{k} 10^{-}$ & 57.67 & 58.28 & 0.999 & 0.999 & 0.996 & 0.8175 \\
\hline $\mathrm{k} 11^{\square}$ & 57.67 & 58.35 & 0.999 & 0.999 & 0.995 & 0.6675 \\
\hline $\mathrm{k} 12^{\text {口 }}$ & 57.67 & 58.35 & 0.999 & 0.999 & 0.992 & 0.4965 \\
\hline $\mathrm{k} 13^{\text {口 }}$ & 57.67 & 58.36 & 0.999 & 0.999 & 0.999 & 0.4989 \\
\hline $\mathrm{k} 14^{\square}$ & 57.67 & 58.38 & 0.999 & 0.999 & 0.999 & 0.7316 \\
\hline $\mathrm{k} 15^{\square}$ & 57.67 & 58.66 & 0.999 & 0.999 & 0.978 & 0.7650 \\
\hline $\mathrm{k} 16^{\square}$ & 57.67 & 58.34 & 0.999 & 0.999 & 0.997 & 0.7067 \\
\hline $\mathrm{k} 17$ & 57.67 & 58.30 & 0.999 & 0.999 & 0.997 & 0.8122 \\
\hline k18 & 57.67 & 58.30 & 0.999 & 0.999 & 0.999 & 0.7226 \\
\hline k19" & 57.67 & 58.30 & 0.999 & 0.999 & 0.998 & 0.8221 \\
\hline $\mathrm{k} 20^{\text {口 }}$ & 57.67 & 58.42 & 0.999 & 0.999 & 0.992 & 0.7273 \\
\hline $\mathrm{k} 21^{\text {ㅁ }}$ & 57.67 & 58.34 & 0.999 & 0.999 & 0.997 & 0.6127 \\
\hline $\mathrm{k} 22^{\square}$ & 57.67 & 58.35 & 0.999 & 0.999 & 0.999 & 0.6608 \\
\hline $\mathrm{k} 23^{\square}$ & 57.67 & 58.34 & 0.999 & 0.999 & 0.995 & 0.7403 \\
\hline $\mathrm{k} 24^{\square}$ & 57.67 & 58.38 & 0.999 & 0.999 & 0.996 & 0.5769 \\
\hline
\end{tabular}

: image size; ${ }^{\square} 341 \times 512 \times 3 ; " 512 \times 341 \times 3$ 


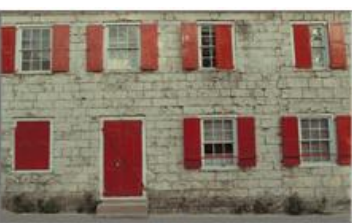

k01

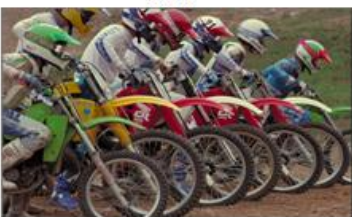

k05

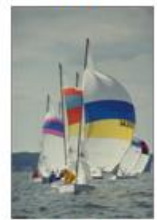

k09

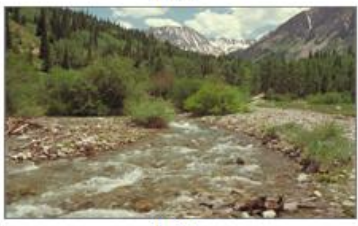

k13

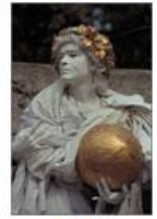

k17

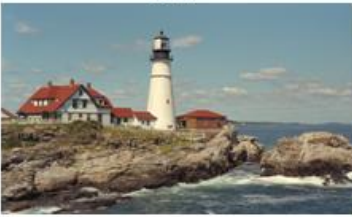

k21

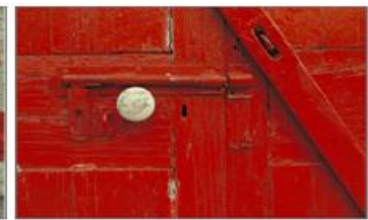

k02

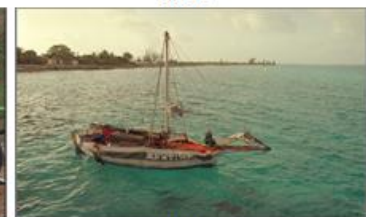

k06

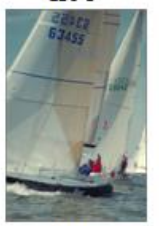

$\mathrm{k} 10$

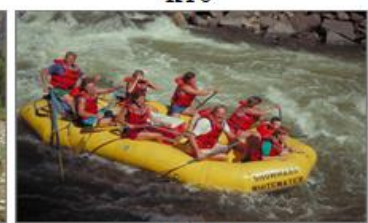

k14

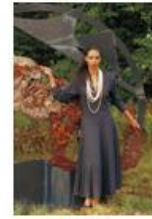

k18

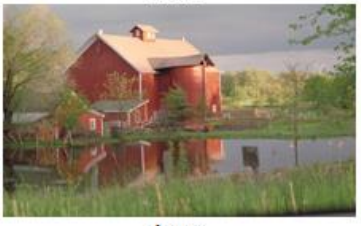

k22

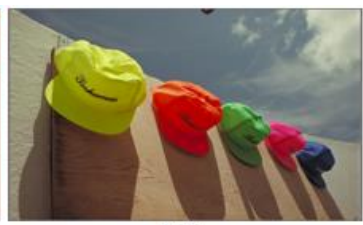

k03

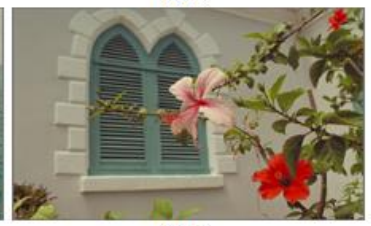

k07

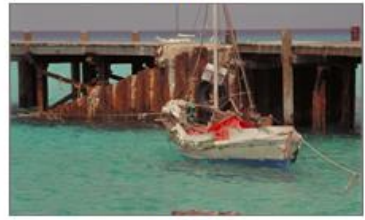

k11

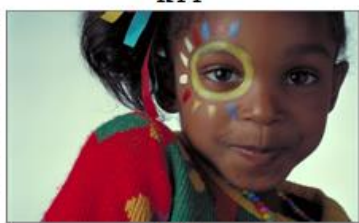

k15

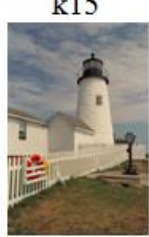

k19

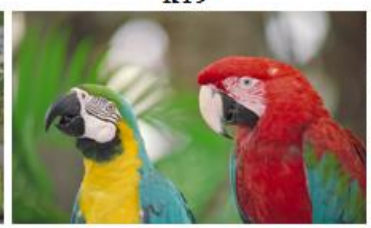

k23

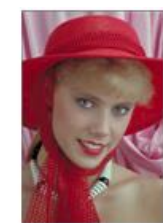

k04

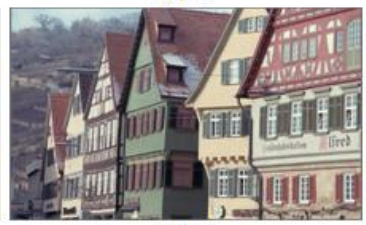

k08

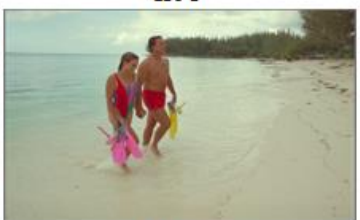

$\mathrm{k} 12$

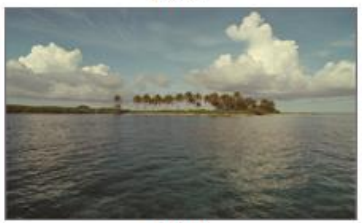

k16

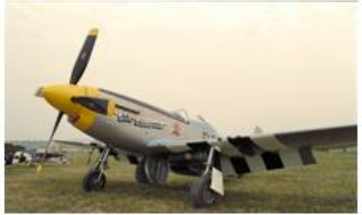

k20

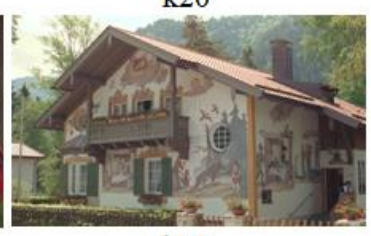

k24

Figure 11. SCISE images used for additional results.

\section{CONCLUSIONS}

Well-known IQA techniques play a significant role in almost all aspects of image processing algorithms. The HVS based technique and algorithm improvements needs are still continue because commonly used IQA techniques cannot properly differentiate the images.

There are two types of ciliary receptor, rod cells and cone cells in the human eye. Rod cells are sensitive to the light and the other one is sensitive to the colors. This means that if you want to get HVS based IQA result, a color image should be separated light and color parts.

In addition, many quality specifications cannot be calculated in time space, but this fact is different in the frequency space. The PIQA uses the color IQA philosophy and the histogram based IQA methodology with the help of the DCT. As a result of these fundamentals, the HSI color model based full-reference perceptual IQA (PIQA) technique is offered in this presented study.

IQA results' ranges are between 0 and 1 as the most IQA techniques. 1 means that top IQA result, 0 means that the worst IQA result for the PIQA. Experimental studies about the reliability of the PIQA on a wellknown test image under different deterioration types, both perceivable by the HVS and with the same PSNR value are given. On the contrary, the PIQA results are more suitable than the others in terms of the HVS and they can be calculated $1.46 \%$ to $2560 \%$ faster than its counterparts' results. Moreover, the PIQA can easily differentiate that impact of deteriorations on colour images. MATLAB ${ }^{\circledR}$ implementation files of the proposed PIQA technique are available online at: shorturl.at/rvAB0 


\section{REFERENCES}

[1] S. E. Umbaugh, "Digital image processing and analysis: human and computer vision applications with CVIPTools," CRC Press; 2011, 2nd edition.

[2] National Geographic, "Image obsessed," National Geographic, p. 35, 2012.

[3] A. C. Bovik, "Automatic prediction of perceptual image and video quality," Proceedings of the IEEE 2013; vol. 101, 2013, pp. 2008-2024.

[4] Y. Yalman, "Histogram based perceptual quality assessment method for color images," Computer Standards \& Interfaces, vol. 36, pp. 899-908, 2014.

[5] Z. Wang, A. C. Bovik and B. Evans, "Blind measurement of blocking artifacts in images," Proceedings of IEEE Int. Conf. Image Process., Canada, 2000, pp. 981-984.

[6] A. C. Bovik and S. Liu, "DCT-domain blind measurement of blocking artifacts in DCT-coded images," Proceedings of IEEE Int. Conf. Acoust. Speech Signal Processing, USA, 2001, pp. 1725-1728.

[7] L. Meesters and J. Martens, "A single-ended blockiness measure for JPEG-coded images," Signal Processing, vol. 82, pp. 369-387, 2002.

[8] S. Liu and A.C. Bovik, "Efficient DCT-domain blind measurement and reduction of blocking artifacts", IEEE Trans. Circuits Syst. Video Technology, vol. 12, pp. 1139-1149, 2002.

[9] Z. Wang and A. C. Bovik, "Mean squared error: Love it or leave it? A new look at signal fidelity measures," IEEE Signal Processing Magazine, vol. 26, pp. 98-117, 2009.

[10] N. Ponomarenko, F. Silvestri, K. Egiazarian, M. Carli and V. Lukin, "On between-coefficient contrast masking of DCT basis functions," 3rd Int. Workshop on Video processing and quality metrics: VPQM '07, USA, 2007, 4p.

[11] Z. Wang, A. C. Bovik, H. R. Sheikh and E. P. Simoncelli, "Image quality assessment: From error measurement to structural similarity," IEEE Transactions on Image Processing, vol. 13, pp. 600-612, 2004.

[12] Z. Wang, E. P. Simoncelli and A. C. Bovik, "Multiscale structural similarity for image quality assessment," Invited Paper, Proceedings of IEEE Asilomar Conference on Signals, Systems and Computers, USA, 2003, pp. 1398-1402.

[13] Z. Wang and A. C. Bovik, "A universal image quality index," IEEE Signal Processing Letters, vol. 9, pp. 81-84, 2002.

[14] H. D. Sheikh and A. C. Bovik, "Image information and visual quality," IEEE Trans. Image Processing, vol. 15, pp. 430-444, 2006

[15] T. Dacheng, L. Xuelong, L. Wen and G. Xinbo, "Reduced-reference IQA in contourlet domain," IEEE Transactions on Systems, Man, and Cybernetics, vol. 39, pp. 1623-1627, 2009.

[16] R. Soundararajan and A. C. Bovik, "Video quality assessment by reduced reference spatio-temporal entropic differencing," IEEE Trans. Circuits Syst. Video Technology, vol. 23, pp. 684-694, 2012.

[17] Z. Wang and A. C. Bovik, "Reduced and no-reference image quality assessment," IEEE Signal Process. Magazine, vol. 28, pp. 29-40, 2011.

[18] Z. Wang and E. P. Simoncelli, "Reduced-reference image quality assessment using a wavelet domain natural image statistic model," Proceedings of SPIEV Int. Soc. Opt. Eng., 2005, pp. 149-159.

[19] Q. Li and Z. Wang, "Reduced-reference image quality assessment using divisive normalization-based image representation,” IEEE J. Sel. Top. Signal Processing, vol. 3, pp. 202-211, 2009.

[20] R. Soundararajan and A. C. Bovik, "RRED indices: Reduced reference entropic differencing for image quality assessment," IEEE Trans. Image Processing, vol. 21, pp. 517-526, 2012.

[21] R. C. Gonzalez and R. E Woods, "Digital Image Processing,” Pearson Prentice Hall, 3rd Edition, 2008.

[22] Y. Yalman and I. Erturk, "A new color image quality measure based on YUV transformation and PSNR for human vision system," Turkish Journal of Electrical Engineering \& Computer Sciences, vol. 21, pp. 603-612, 2013.

[23] S. H. Schwartz, "Visual Perception: A Clinical Orientation," McGraw-Hill, 4th Edition, 2010.

[24] V. Bruce, P.R. Green and M. A. Georgeson, "Visual Perception: Physiology, Psychology \& Ecology," Psychology Press, Taylor \& Francis Group, 4th Edition 2010.

[25] S.J. Williamson and H.Z. Cummins, "Light and Color in Nature and Art," Wiley, 1983.

[26] Y. Yalman, “A histogram based image quality index," Przeglad Elektrotechniczny, vol. 88, pp. 126-129, 2012.

[27] A. M. Eskicioglu and P. S. Fisher, "Image quality measures and their performance," IEEE Transactions on Communications, vol. 43, pp. 2959-2965, 1995.

[28] M. Rabbani and P. W. Jones, "Digital image compression techniques," SPIE Optical Engineering Press, Washington, 1991.

[29] 'Rochester Institute of Technology, Rods \& Cones', http://www.cis.rit.edu/people/faculty/montag/ vandplite/pages/chap_9/ch9p1.html, [accessed 18 January 2020]

[30] S. J. Williamson and H. Z. Cummins, "Light and Color in Nature and Art," John Wiley and Sons, 1983.

[31] 'Stanford Center for Image Systems Engineering', https://scien.stanford.edu/index.php/test-images-and-videos/, [accessed 19 January 2020]

\section{BIOGRAPHY OF AUTHOR}

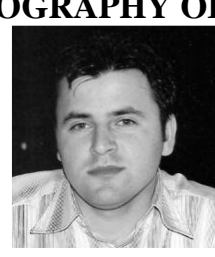

Yildiray Yalman, received the M.Sc. and Ph.D. degree from Kocaeli University, Turkey, in 2007 and 2010, respectively. He is currently working as a Professor at Piri Reis University in Turkey. His active research interests are data hiding, steganography, image processing and real-time multimedia communications. 